\title{
Interaction and cellular uptake of surface-modified carbon dot nanoparticles by J774.1 macrophages
}

\author{
LESTER THOO ${ }^{I}$, MOCHAMAD Z. FAHMI', IHSAN N. ZULKIPLII ${ }^{1}$, NATASHA KEASBERRY', \\ $A D I I D R I S^{l}$
}

${ }^{1}$ PAPRSB Institute of Health Science, Universiti Brunei Darussalam, Brunei Darussalam

${ }^{2}$ Department of Chemistry, Universitas Airlangga, Indonesia

${ }^{3}$ Faculty of Science, Universiti Brunei Darussalam, Brunei Darussalam

\begin{abstract}
Carbon dot (Cdot) nanoparticles are an emerging class of carbon nanomaterials with a promising potential for drug delivery and bio imaging applications. Although the interaction between Cdots and non-immune cell types has been well studied, Cdot interactions with macrophages have not been investigated. Exposure of Cdot nanoparticles to J774.1 cells, a murine macrophage cell line, resulted in minimal toxicity, where notable toxicity was only seen with Cdot concentrations higher than $0.5 \mathrm{mg} /$ $\mathrm{ml}$. Flow cytometric analysis revealed that $C$ dots prepared from citric acid were internalized at significantly higher levels by macrophages compared with those prepared from bamboo leaves. Interestingly, macrophages preferentially took up phenylboronic acid (PB)-modified nanoparticles. By fluorescence microscopy, strong blue light-specific punctate Cdot fluorescence resembling Cdot structures in the cytosolic space was mostly observed in J774.1 macrophages exposed to PB-modified nanoparticles and not unmodified Cdot nanoparticles. $P B$ binds to sialic acid residues that are overexpressed on diseased cell surfaces. Our findings demonstrate that PB-conjugated Cdots can be taken up by macrophages with low toxicity and high efficiency. These modified Cdots can be used to deliver drugs to suppress or eliminate aberrant immune cells such as macrophages associated with tumors such as tumor-associated macrophages.
\end{abstract}

Key words: carbon dots, macrophage, nanoparticles, phenylboronic acid, J774.1 cells.

(Centr Eur Immunol 2017; 42 (2): 324-330)

\section{Introduction}

Cdot nanoparticles are an emerging group of fluorescent nanomaterials or quantum dots with excellent photoluminescence and biocompatibility [1]. These water-soluble nanoparticles exert minimal cellular toxicity because they do not contain toxic heavy metals unlike their other fluorescent counterparts, quantum dots and some organic dyes [2]. Cdots have the ability to emit specific wavelengths of light, which resembles the optical properties of conventional quantum dots [3]. Furthermore, Cdots are relatively easy and inexpensive to synthesize and subject to surface modifications. Generally, Cdots consist of carbon, hydrogen and oxygen, and their carbon configuration with a quasi-spherical form has a promising potential in a number of applications such as probes for bio imaging and in optoelectronic and biosensor technologies [4].
Cdots have also been tested for the delivery of therapeutic agents. Recent studies have shown that Cdots can be used to deliver nucleic acids into target cells for gene therapy, both in vivo and in vitro [5-9]. Furthermore, Cdots have been applied to intracellular drug delivery [10-12]. However, in vitro studies to date have only tested the effects of Cdot exposure on non-immune cell types. It is possible that Cdot toxicity and cellular uptake may vary in other cell types, which is important because different mechanisms of nanoparticle uptake may dictate the mode that the loaded "cargo" is processed by the cells. In this study, we examined how Cdots interact with macrophages, an immune cell type that has an exceptional ability to take up extracellular particulate matter such as nanoparticles. Subsequently, we explored the interactions between various Cdot preparations with different surface modifications and J744.1 cells, a murine macrophage cell line, by assessing Cdot cytotoxicity and uptake efficiency. There is

Correspondence: Adi Idris, PAPRSB Institute of Health Science, Universiti Brunei Darussalam Jalan Tungku Link Gadong, BE1410 Bandar Seri Begawan, Brunei Darussalam, e-mail: yusri.idris@ubd.edu.bn Submitted: 23.03.2016; Accepted: 13.06.2016 
growing interest in taking advantage of sialic acid (SA), which is expressed highly on cell surfaces in disease states (e.g. oncogenic transformation) [13], as a "tag" for diseased cells. It is known that phenylboronic acid (PB) reacts strongly with SA residues with high affinity and specificity [14]. This property makes PB an ideal ligand that allows highly specific and efficient labeling of SA residues on cells. We therefore functionalized the surface of the Cdots with PB groups in our study to determine whether this modification enhances cellular uptake of Cdots.

In this study, we found that Cdots exerted low toxicity in J774.1 macrophages, even at high amounts. Flow cytometry and fluorescence microscopy revealed that J774.1 macrophages internalized citric acid (CA)-derived Cdots more efficiently than Cdots prepared from an alternative source of carbon, cellulose from bamboo leaf (BA). Specifically, J774.1 macrophages showed high efficiency for taking up PB-modified CA-derived Cdots.

\section{Material and methods}

\section{Chemicals}

4',6-Diamidine-2'-phenylindole dihydrochloride (DAPI) was obtained from Invitrogen (Grand Island, NY). Alexa Fluor® 647 Phalloidin (Cat\# A22287) was obtained from Molecular Probes (Waltham, MA). 3-(4,5-Dimethylthiazol-2-yl)-2,5-diphenyl tetrazolium bromide (MTT) and carboxyl phenylboronic acid (CBBA) were obtained from Sigma-Aldrich (St. Louis, MO).

\section{Preparation of Cdots}

Cdots were prepared by pyrolyzing either CA or BA. For nanoparticles prepared from CA, $200 \mathrm{mg}$ CA was calcined at $240^{\circ} \mathrm{C}$ for $30 \mathrm{~min}$ before cooling to room temperature. This process turned CA into an orange color, owing to carbonization and the generation of graphitic nanostructures. For nanoparticles prepared from BA, 200 $\mathrm{mg}$ of sliced bamboo leaves were refluxed with $10 \mathrm{ml}$ of $2 \% \mathrm{NaOH}$ for $5 \mathrm{~h}$. After washing with water, cellulose was mixed with $10 \mathrm{ml}$ of $5 \% \mathrm{NaOCl}$ using a magnetic stirrer for $2 \mathrm{~h}$ and then centrifuged at $700 \mathrm{~g}$ for $5 \mathrm{~min}$. To pyrolyze the cellulose, $200 \mathrm{mg}$ cellulose was calcined at $540^{\circ} \mathrm{C}$ for $30 \mathrm{~min}$ before cooling to room temperature. After calcination of CA- and BA-prepared nanoparticles, $0.5 \mathrm{M}$ $\mathrm{NaOH}$ was added until the $\mathrm{pH}$ became neutral. To narrow the size distribution of the nanoparticles and remove small molecules, nanoparticles were passed through centrifugal filter units with a $1 \mathrm{kDa}$ molecular weight cut-off.

\section{Conjugating CBBA to Cdots}

$10 \mathrm{ml}$ of CBBA solution was prepared by adding 2.4 mg 4-dimethylaminopyrid to a $0.3 \mathrm{mg} / \mathrm{ml} \mathrm{CBBA}$ solution before adding $3.1 \mathrm{mg}$ of 1-ethyl-3-(3-dimethylaminopropyl) carbodiimide. After mixing for $30 \mathrm{~min}, 5 \mathrm{ml}$ of CA- or BA-prepared nanoparticles was added to the mixture, followed by stirring for $24 \mathrm{~h}$. The mixture then underwent dialysis for $24 \mathrm{~h}$.

\section{J774.1 macrophage culture}

J774. 1 cells were obtained from the American Type Tissue Culture Collection (Manassas, VA). Cells were cultured in complete medium consisting of RPMI 1640 medium supplemented with $10 \%$ fetal calf serum, $2 \mathrm{mM} \mathrm{L-glu-}$ tamine, $20 \mathrm{U} / \mathrm{ml}$ penicillin, and $20 \mu \mathrm{g} / \mathrm{ml}$ streptomycin (all purchased from Gibco, Carlsbad, CA) and grown on bacteriological plastic (Sterilin, Newport, United Kingdom).

\section{Exposure of Cdots to J774.1 macrophages}

Cells $\left(4 \times 10^{5}\right.$ cells $)$ were seeded in 12 -well or 96 -well plates (Corning, Tewksbury, MA), incubated overnight, and then treated with or without various concentrations $(5 \mathrm{mg} / \mathrm{ml}$ to $5 \mathrm{ng} / \mathrm{ml}$ ) of different Cdot preparations previously dissolved in sterile water, for $24 \mathrm{~h}$ before performing MTT assays. Sterile water was used as the vehicle control. For microscopic analysis of internalized Cdots, cells $\left(4 \times 10^{5} /\right.$ well $)$ were seeded on autoclaved glass coverslips in 12-well plates (Corning, Tewksbury, MA), incubated overnight, and then treated with nanoparticles.

\section{Assessment of Cdot cytotoxicity in J774.1 macrophages}

An MTT assay was performed to measure cell viability. After $24 \mathrm{~h}$, cell medium was replaced with a solution of $1 \mathrm{mg} / \mathrm{ml}$ MTT in phosphate-buffered saline (PBS), followed by incubation for $1 \mathrm{~h}$ at $37^{\circ} \mathrm{C}$. After removing the MTT solution, $200 \mu \mathrm{l}$ of a $50 \%$ isopropanol/10\% SDS solution was added to each well, followed by incubation in the dark overnight. The absorbance of the wells was measured spectrophotometrically at $570 \mathrm{~nm}$ on a BioTek ELx808 ELISA plate reader (BioTek, Winooski, VT).

\section{Measurement of cellule uptake of Cdots by flow cytometry}

At $24 \mathrm{~h}$ post-treatment with Cdots, the medium was discarded and the cells were rinsed with PBS. Cell monolayers were fixed in situ for $5 \mathrm{~min}$ with $4 \%$ (w/v) paraformaldehyde (PFA) in PBS and then rinsed with PBS. PBS $(250 \mu \mathrm{l})$ was added to the cells before carefully scraping the cells off the plate. The cells were transferred into clean tubes for intracellular fluorescence analysis on a BD Accuri ${ }^{\circledR}$ C6 Flow Cytometer (BD Bioscience, San Jose, CA).

\section{Immunofluorescence staining and microscopic visualization of internalized Cdots}

At $24 \mathrm{~h}$ post-treatment with Cdots, the medium was discarded and the cells were rinsed with PBS. Cell monolayers on glass coverslips were then fixed in situ for $5 \mathrm{~min}$ 
Table 1. Cdots used in this study

\begin{tabular}{lc}
\hline Cdots & Abbreviation \\
\hline Cdots prepared from citric acid & $\mathrm{CDA}$ \\
\hline $\begin{array}{l}\text { Boronic acid-conjugated Cdots prepared } \\
\text { from citric acid }\end{array}$ & $\mathrm{CDA} / \mathrm{CBBA}$ \\
\hline Cdots prepared from bamboo leaf & $\mathrm{CDB}$ \\
\hline $\begin{array}{l}\text { Boronic acid-conjugated Cdots prepared } \\
\text { from bamboo leaf }\end{array}$ & $\mathrm{CDB} / \mathrm{CBBA}$ \\
\hline
\end{tabular}

with $4 \%(w / v)$ PFA and $0.1 \%$ Triton X-100 in PBS. After rinsing with PBS, the cells were incubated with Alexa Fluor® 647 Phalloidin at a dilution of $1 / 40$ in PBS for 20 min at room temperature in the dark and then rinsed three times with PBS. The cells were washed once in PBS and then stained with $10 \mathrm{ng} / \mathrm{mL}$ DAPI in PBS for $10 \mathrm{~min}$ in the dark. After washing in PBS, the cells were mounted with DakoCytomation florescence mounting medium (Dako, Glostrup, Denmark) under a coverslip. The cells were then analyzed using an epi-fluorescence microscope (Nikon inverted microscope Eclipse 90L) at 100× magnification.

\section{Results}

\section{Physiochemical properties of Cdots}

Spectroscopic characterization and microscopy were carried out to determine the physiochemical properties of the prepared Cdots (Table 1). The PL spectra for both CDA and $\mathrm{CDB}$ showed that the particles exhibited fluorescence emission at $480 \mathrm{~nm}(\lambda \mathrm{ex}=420 \mathrm{~nm})$ (Fig. 1A), similar to Cdots reported by Zakki et al. [12]. Although some larger particles were observed (above $15 \mathrm{~nm}$ ), AFM analysis revealed the average size distribution for the majority of CDA and CDB nanoparticles to be $3 \mathrm{~nm}$ and $5 \mathrm{~nm}$, respectively (Fig. 1B). Raman spectroscopy confirmed that CDA and CDB nanoparticles had similar Raman spectral patterns (Fig. 1C). When compared to the raman spectra of $\mathrm{CA}$ or BA alone, the raman absorption profiles of CDA and CDB demonstrated similar characteristics. Both had peaks at 1332 and $1582 \mathrm{~nm}$ (D and G bands respectively) which correlate to sp2- and sp3-hybridized carbon atoms, respectively. These findings confirm that the prepared Cdots had the expected characteristics, which were similarly shared by CDA and CDB nanoparticles.
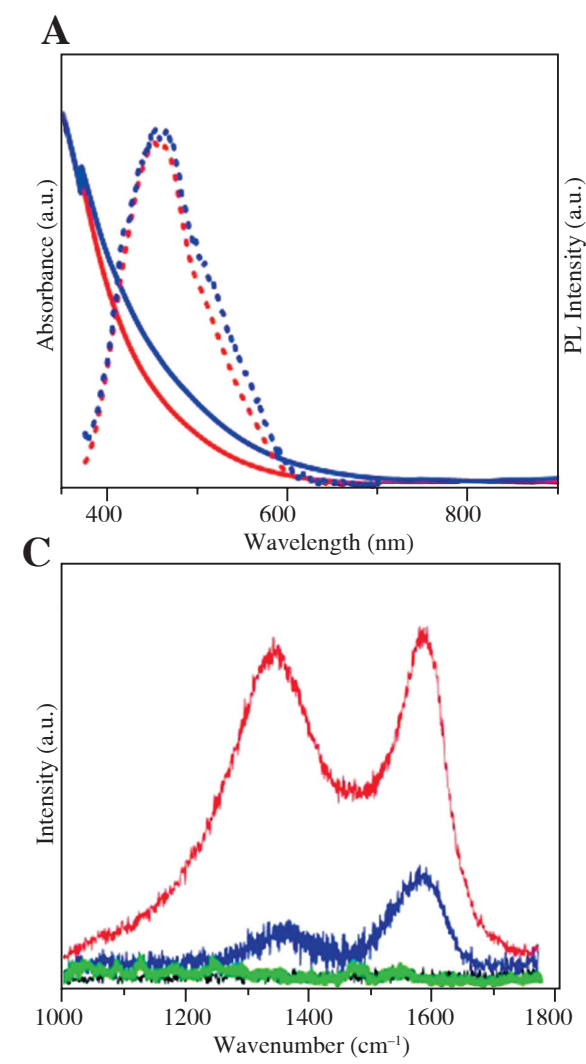

B
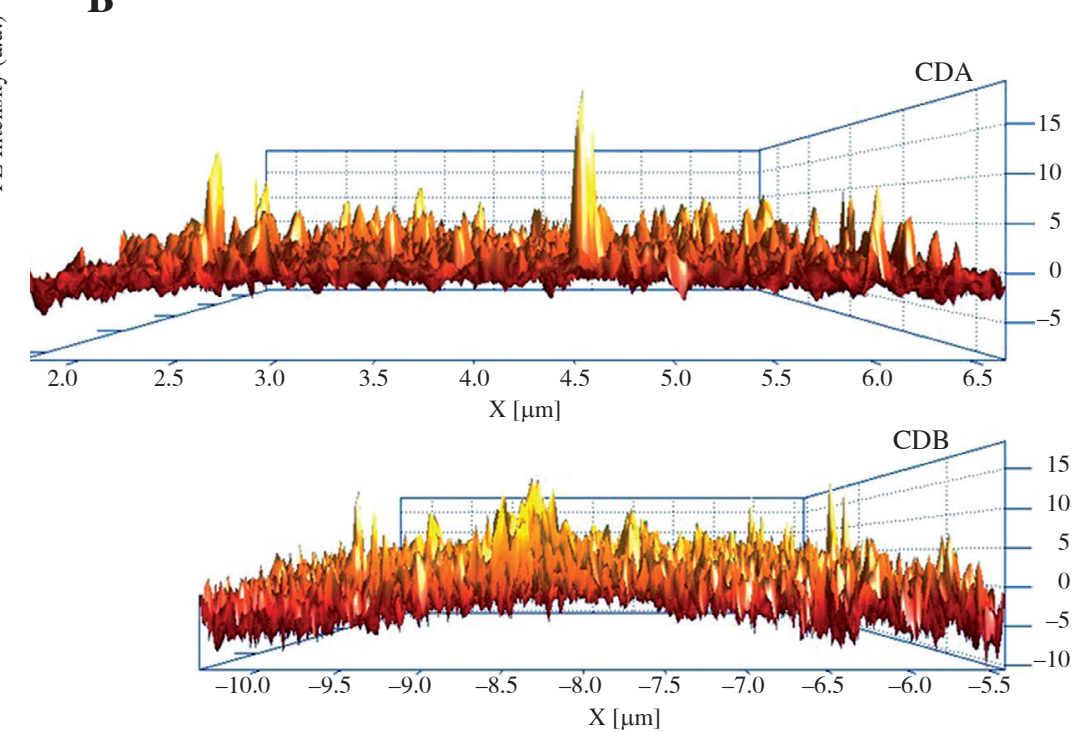

Fig. 1. Physicochemical properties of Cdots used in this study. A) UV-Vis absorption spectra (solid line) and PL emission spectra (dotted line) of prepared CDA (red) and CDB (blue). The sample was measured at $\lambda \mathrm{ex}=420 \mathrm{~nm}$. B) AFM characterization of CDA and CDB on the cross-sectional side of the samples. C) Raman spectra of CA (black), BA (green), CDA (red) and CDB (blue) samples 


\section{Cdots have minimal toxicity in $\mathbf{J 7 7 4 . 1}$ macrophages}

The toxicity of Cdots in vitro has only been tested in non-immune cell lines such as HeLa [12, 15], MCF-7 [5], MDA-MB231 [5], Vero [15], and L929 [16]. Therefore, we tested the toxicity of Cdots in J774.1 macrophages after $24 \mathrm{~h}$ of exposure to various Cdot preparations using the MTT assay, a colorimetric assay that measures the metabolic activity of cells. Compared with the vehicle control (water), cells treated with increasing doses of Cdots maintained high viability (>90\% viability) (Fig. 2). Notable toxicity was only seen at higher Cdot concentrations $(0.5-5 \mathrm{mg} / \mathrm{ml})$. With the exception of CDB, treatment with other Cdot preparations only resulted in less than $50 \%$ percent loss of cell viability at the highest dose $(5 \mathrm{mg} / \mathrm{ml})$ compared with water treatment alone. Overall, we found that Cdots exerted minimal toxicity in J774.1 macrophages and that the optimal dose for subsequent Cdot uptake experiments was $0.05 \mathrm{mg} / \mathrm{ml}$.

\section{CDA/CBBA is internalized most efficiently by J774.1 macrophages}

Upon exposure to particulate matter such as nanoparticles, macrophages are an important cell type for elicitation of innate immune responses and the destruction and/or clearance of particles from tissues. Prior to these events, macrophages internalize the particles. To assess the efficiency of Cdot uptake by J774.1 macrophages, various Cdot preparations were exposed to adherent cells for $24 \mathrm{~h}$ to allow the Cdots to be internalized before analyzing the cells by flow cytometry and fluorescence microscopy. The excitation-dependent fluorescence of Cdots (blue light; $\lambda$ $=488 \mathrm{~nm}$ ) allowed visualization of Cdots intracellularly. Compared with the control, J774.1 macrophages internalized significantly more CA-derived Cdots (CDA and CDA/ CBBA) than BA-derived Cdots ( $\mathrm{CDB}$ and $\mathrm{CDB} / \mathrm{CBBA})$ $(p<0.05)$ (Fig. 3A and B). Although not statistically significant, J774.1 cells exposed to the PB-modified form of CDA, CDA/CBBA, had a higher mean fluorescence intensity compared with cells exposed to CDA (Fig. 3B). Nonetheless, the percentage uptake of CDA/CBBA was markedly higher than that of CDA (Fig. 3A). These findings suggest that J774.1 macrophages internalize CDA nanoparticles more efficiently than $\mathrm{CDB}$ nanoparticles, and that surface PB-modification appears to enhance the uptake of CDA nanoparticles.

\section{Internalized CDA/CBBA appear as "punctate dots" in the cytosol}

Flow cytometric analysis showed that more PB-modified CDA nanoparticles were taken up by cells compared with non-PB-modified CDA nanoparticles (Fig. 3A). To confirm this result, we performed fluorescence microscop- ic analysis of the internalized Cdots at $24 \mathrm{~h}$ post-exposure. Strong blue light-specific punctate Cdot fluorescence resembling Cdot structures in the cytosolic space was mostly observed in J774.1 macrophages exposed to CDA/CBBA nanoparticles and not unmodified CDA or other Cdot nanoparticles (Fig. 4). This finding demonstrates that J774.1 macrophages preferentially take up CDA/CBBA nanoparticles compared with the other tested Cdots.

\section{Discussion}

Initially, we performed physiochemical studies to ensure that the prepared Cdots had the correct characteristics. The UV spectra of Cdots is mostly attributed by $\varpi-\varpi^{*}$ transitions in the aromatic sp2 carbon atom domains [12, 17]. The emission results from the formation of carbon crystals on the graphene-like structure and the expansion of the orbital state due to the $\mathrm{sp} 2$ and $\mathrm{sp} 3$ carbon structures, which drive on discrete quantized orbital energy levels [18]. Although the UV spectral patterns for CDA and $\mathrm{CDB}$ were similar, AFM analysis revealed that the nanoparticle sizes between CDA and CDB slightly differ (e.g. $3 \mathrm{~nm}$ for CDA and $5 \mathrm{~nm}$ for $\mathrm{CDB}$ ). Moreover, the pyrolysis process of citric acid yields CDA particles which are relatively smaller than $\mathrm{CDB}$. It is possible that during the pyrolysis process of CA, CA molecules were re-arranged to form larger structures in CDA and leading to its smaller size distribution than CDB. Raman spectroscopy revealed sp2 (D band) and sp3 (G band) carbon structures

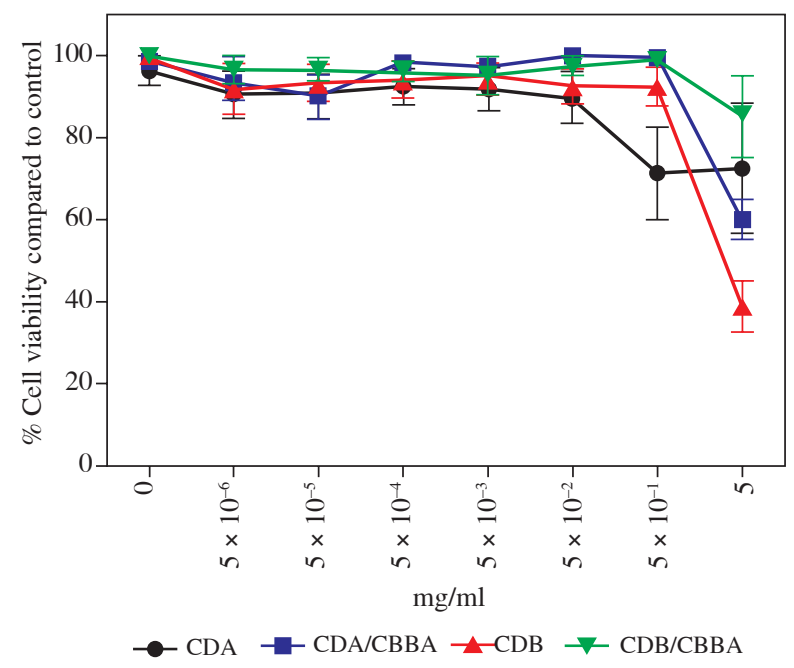

Fig. 2. Minimal toxicity of Cdots in J774.1 cells. J774.1 cells were incubated with various concentrations of different Cdot preparations for $24 \mathrm{~h}$ before measuring cell viability by MTT assays. Percentage cell viability was determined by comparing the effect of Cdots with the vehicle control (water). Data represent the mean \pm SEM of four independent experiments 

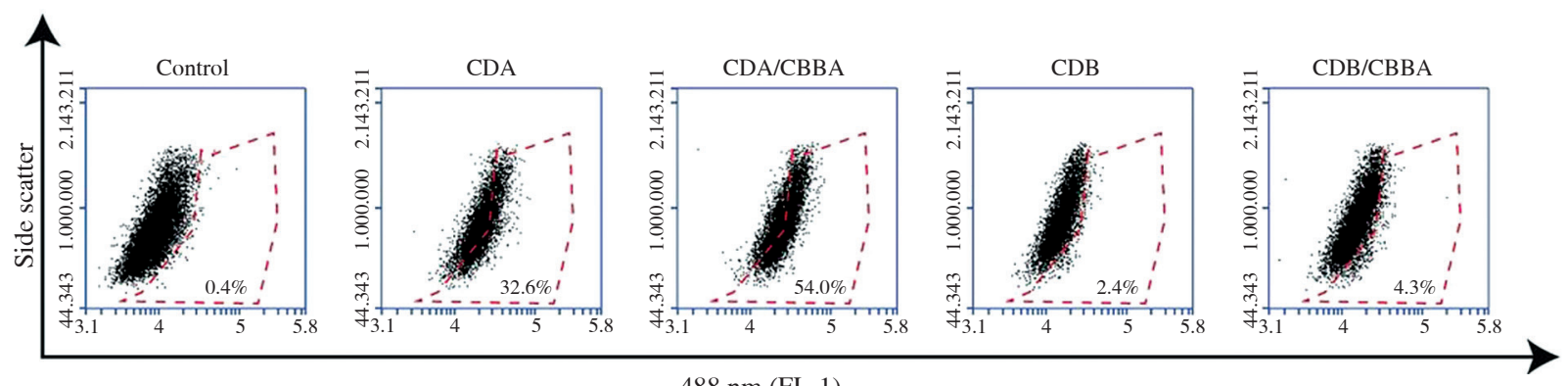

$488 \mathrm{~nm}$ (FL-1)

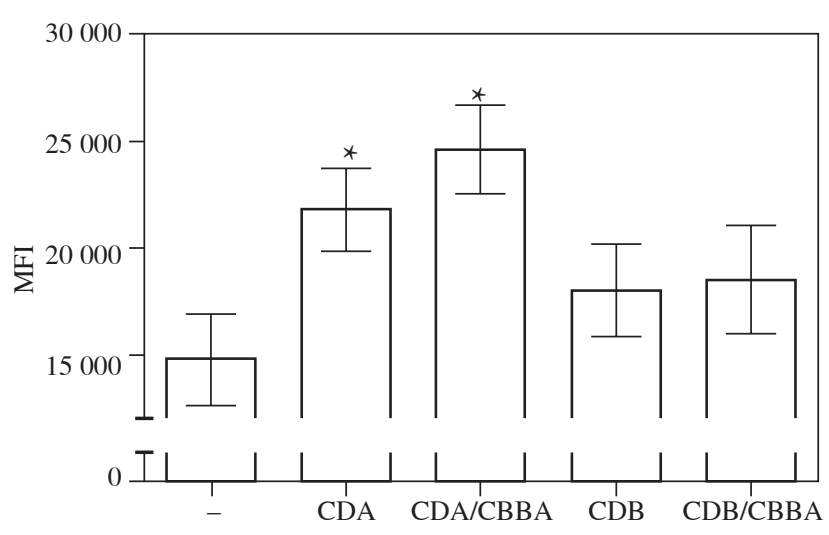

Fig. 3. J774.1 cells internalize CDA/CBBA nanoparticles more efficiently compared with other Cdot nanoparticles. J774. 1 cells were incubated with $0.05 \mathrm{mg} / \mathrm{ml}$ of various Cdot preparations or water (-) for $24 \mathrm{~h}$ before A) measuring the percentage of Cdot uptake $(488 \mathrm{~nm}$ ) by flow cytometry. Results are representative of four independent experiments. B) Examples of (A) are shown with graphs showing the mean fluorescence intensity (MFI) \pm SEM from four independent experiments; ${ }^{*} p<0.05$, Student's $t$ test

which suggests the presence of graphene-like structures. The presence of these bands in Cdots further supports the sp2 graphitic-defect sites in sp3 amorphous carbon, which is responsible for its photo luminescent properties.

In this study, we produced water-soluble Cdot nanoparticles conjugated with PB groups on their surface. As mentioned previously, PB has a high affinity for glycoproteins such as SA that is overexpressed on a wide variety of pathological cells [13]. Because PB groups can be used to target SA ligands on diseased cells, PB-conjugated Cdots could potentially be used as molecular imaging probes and drug delivery agents targeting diseased cells such as tumor cells. Our observation that Cdots exert minimal toxicity in J774.1 cells corroborates previous findings in other cell types $[5,12,15]$. To our knowledge, this is the first study to investigate the interaction between Cdot nanoparticles and macrophages. A previous study by Qin et al. [19] showed that exposure of human macrophages to graphene quantum dots, a carbon material family member, results in apoptotic cell death and production of reactive oxygen species. Our findings suggest that Cdots could be used as a non-toxic alternative to graphene quantum dots for a number of applications.

We found that J774.1 macrophages took up significantly more CA-prepared Cdots than BA-prepared Cdots. This result suggests that the source of carbon for preparing Cdot dictates the uptake efficiency by macrophage, possibly due to the differences in the surface chemistry of the Cdots. Cellular uptake is generally influenced by several properties including size, shape and surface charge of nanoparticles [20]. It is possible the surface charge on the Cdots may vary depending on the carbon source. Future work is required to assess the surface charge of Cdots by dynamic light scattering (DLS) analysis. Nanoparticles are mainly internalized into phagocytic cells such as macrophages via fluid phase endocytosis, receptor-mediated endocytosis, and phagocytosis [21]. The uptake mechanism of Cdots by macrophages is not yet understood. Future studies to elucidate this mechanism are crucial to understand the detailed dynamics of the Cdot internalization process. It is interesting to note that PB-coated CDA was more efficiently taken up by macrophages. The murine J774.1 macrophage cell line was originally derived from a tumor (reticulum cell 


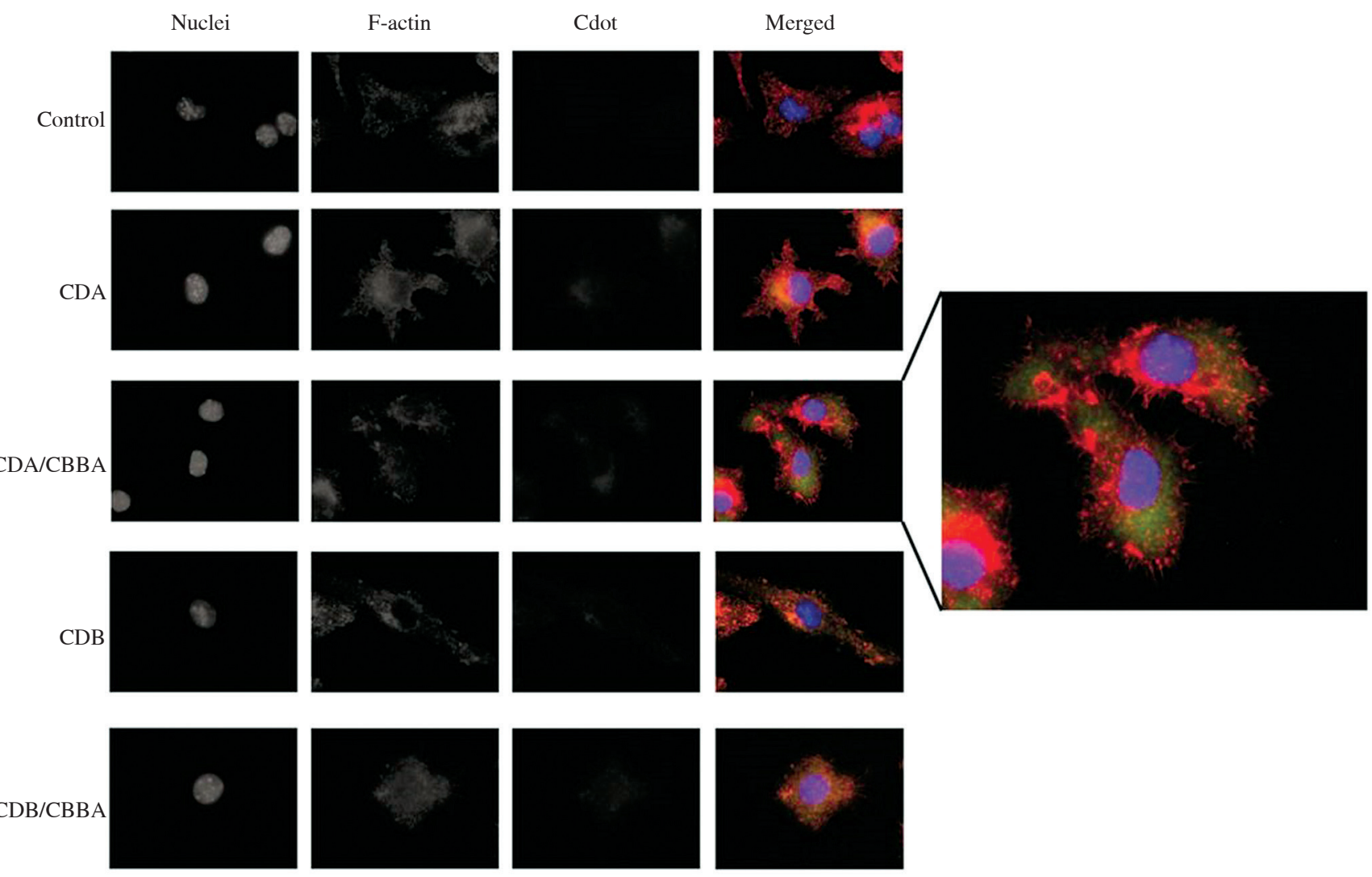

Fig. 4. Internalized CDA/CBB nanoparticles are localized in the cytosolic space. J774.1 cells were incubated with 0.05 $\mathrm{mg} / \mathrm{ml}$ of various Cdot preparations or water (-) for $24 \mathrm{~h}$ before fixing the cells for immunofluorescence staining and microscopic analysis of internalized Cdots. Cell nuclei (blue) and filamentous actin (red) were stained with DAPI and Phalloidin-647, respectively, before observing under a fluorescence microscope at $\times 100$ magnification. Cdot fluorescence was detected at a wavelength of $488 \mathrm{~nm}$ (green). Data are representative of four independent experiments

carcinoma)-bearing mouse [22]. Considering its cancerous (i.e. diseased) origin, it is possible that SA groups are highly expressed on the cell surface of J774.1 macrophages, and therefore preferentially internalize PB-modified Cdots. However, we have not demonstrated this experimentally. To confirm this, future work will assess the cellular uptake of Cdots using macrophages treated with sialidase, which is used to cleave sialic acid residues from the cells, or using non-treated macrophages cultured in the presence of free PBA, as performed previously on murine melanoma cells [23]. Furthermore, Cdot uptake experiments using human and murine primary macrophages will be necessary to definitively elucidate macrophage interactions with Cdots in a non-diseased state.

Cell-specific imaging to target specific cell populations for diagnosis (e.g. tumor cells) and eventual cell-directed therapy is critical to attain high therapeutic success. In cancer, abnormal sialylation is a characteristic associated with malignant properties including invasiveness and metastatic potential [24]. Therefore, alterations associated with sialylation facilitate early diagnosis, prognosis, and post-treatment monitoring of various cancers. There is increasing interest to develop PB-functionalized nanocarriers for specific targeting of SA groups to improve drug delivery in cancer therapy [25]. Considering that Cdots have a promising potential for bio imaging [4], Cdots bearing PB surface modifications could serve as a good nanocarrier candidate for drug delivery into SA-expressing cells (i.e. diseased cells). Future study will focus on elucidating the mechanism of Cdot uptake by phagocytic cells such as macrophages. Furthermore, the exact mechanism of in vivo bio distribution, cellular uptake, and long-term toxicological effects of Cdots remain to be investigated.

This study represents an advance in the field of fluorescent nanomaterial research. Our findings demonstrate that PB-conjugated Cdots can be taken up into macrophages in vitro with low toxicity and high efficiency for a number of potential biological applications such as intracellular tracking of internalized Cdot-coupled antigens and drug/ vaccine delivery for cellular immunotherapy. Importantly, 
macrophage-associated delivery of drugs can be used to suppress or eliminate macrophages associated with tumors (i.e. tumor-associated macrophages) [26].

This work was supported by the Southeast and South Asia and Taiwan Universities (SATU) Joint Research Scheme.

The authors declare no conflict of interest.

\section{References}

1. Wang J, Liu G, Cham-Fai Leung K, Loffroy R, et al. (2015): Opportunities and Challenges of Fluorescent Carbon Dots in Translational Optical Imaging. Current pharmaceutical design 21: 5401-5416.

2. Resch-Genger U, Grabolle M, Cavaliere-Jaricot S, et al. (2008): Quantum dots versus organic dyes as fluorescent labels. Nat Met 5: 763-775.

3. Fu M, Ehrat F, Wang Y, et al. (2015): Carbon dots: a unique fluorescent cocktail of polycyclic aromatic hydrocarbons. Nano Lett 15: 6030-6035.

4. Shi H, Wei J, Qiang L, et al. (2014): Fluorescent carbon dots for bioimaging and biosensing applications. J Biomed Nanotech 10: 2677-2699.

5. Misra SK, Ohoka A, Kolmodin NJ, et al. (2015): Next generation carbon nanoparticles for efficient gene therapy. Mol Pharm12: 375-385.

6. Pierrat P, Wang R, Kereselidze D, et al. (2015): Efficient in vitro and in vivo pulmonary delivery of nucleic acid by carbon dot-based nanocarriers. Biomaterials 51: 290-302.

7. Wang L, Wang X, Bhirde A, et al. (2014): Carbon-dot-based two-photon visible nanocarriers for safe and highly efficient delivery of siRNA and DNA. Adv Healthc Mater 3:12031209.

8. Liu C, Zhang P, Zhai X, et al. (2012): Nano-carrier for gene delivery and bioimaging based on carbon dots with PEI-passivation enhanced fluorescence. Biomaterials 33: 3604-3613.

9. Das S, Debnath N, Cui Y, et al. (2015): Chitosan, Carbon Quantum Dot, and Silica Nanoparticle Mediated dsRNA Delivery for Gene Silencing in Aedes aegypti: A Comparative Analysis. ACS Appl Mater Interfaces 7: 19530-19535.

10. Zhou L, Li Z, Liu Z, Ren J, et al. (2013): Luminescent carbon dot-gated nanovehicles for $\mathrm{pH}$-triggered intracellular controlled release and imaging. Langmuir 29: 6396-6403.

11. Patra S, Roy E, Madhuri R, et al. (2016): The next generation cell-penetrating peptide and carbon dot conjugated nano-liposome for transdermal delivery of curcumin. Biomater Sci 4: 418-429.

12. Fahmi MZ, Chen JK, Huang CC, et al. (2015): Phenylboronic acid-modified magnetic nanoparticles as a platform for carbon dot conjugation and doxorubicin delivery. J Materials Chemistry B 3 (27): 5532-5543.

13. Varki A (2008): Sialic acids in human health and disease. Trends Molecular Med 14: 351-360.

14. Liu A, Peng S, Soo JC, et al. (2010): Quantum dots with phenylboronic acid tags for specific labeling of sialic acids on living cells. Anal Chem 83: 1124-1130.

15. Dekaliuk M, Pyrshev K, Demchenko A (2015): Visualization and detection of live and apoptotic cells with fluorescent carbon nanoparticles. J Nanobiotechnology 13: 86.
16. Du F, Ming Y, Zeng F, et al. (2013): A low cytotoxic and ratiometric fluorescent nanosensor based on carbon-dots for intracellular $\mathrm{pH}$ sensing and mapping. Nanotechnology 24: 365101.

17. Shi QQ, Li YH, Xu Y, et al. (2014): High-yield and high-solubility nitrogen-doped carbon dots: formation, fluorescence mechanism and imaging application. RSC Advances 4: 15631566.

18. Purbia R, Paria S (2016): A simple turn on fluorescent sensor for the selective detection of thiamine using coconut water derived luminescent carbon dots. Biosens Bioelectron 79: 467-475.

19. Qin Y, Zhou ZW, Pan ST, et al. (2015): Graphene quantum dots induce apoptosis, autophagy, and inflammatory response via p38 mitogen-activated protein kinase and nuclear factor- $\kappa \mathrm{B}$ mediated signaling pathways in activated THP-1 macrophages. Toxicology 327: 62-76.

20. Fröhlich E (2012): The role of surface charge in cellular uptake and cytotoxicity of medical nanoparticles. Int J Nanomedicine 7: 5577-5591.

21. Roy R, Kumar S, Tripathi A, et al. (2014): Interactive threats of nanoparticles to the biological system. Immunol Lett 158: 79-87.

22. Ralph P, Nakoinz I (1975): Phagocytosis and cytolysis by a macrophage tumour and its cloned cell line. Nature 257: 393-394.

23. Deshayes S, Cabral H, Ishii T, et al. (2013): Phenylboronic acid-installed polymeric micelles for targeting sialylated epitopes in solid tumors. J Am Chem Soc 135: 15501-15507.

24. Vajaria BN, Patel KR, Begum R, et al. (2015): Sialylation: an Avenue to Target Cancer Cells. Pathol Oncol Res 22: 443447.

25. Wang J, Wu W, Jiang X (2015): Nanoscaled boron-containing delivery systems and therapeutic agents for cancer treatment. Nanomedicine 10: 1149-1163.

26. Vinogradov S, Warren G, Wei X (2014): Macrophages associated with tumors as potential targets and therapeutic intermediates. Nanomedicine 9: 695-707. 